\section{Explicaciones no atendidas sobre las brechas de género en la enfermedad cardiovascular ¿Cómo entender diferencias de género en el infarto de miocardio?}

MARÍA PÍA MARTÍNEZ MARÍN²,a, ISMAEL GALLARDO CUADRA

\section{Unattended explanations on gender gaps in acute myocardial infarction. How can we understand gender gaps in infarction?}

Advances in cardiovascular diseases research have shown that there are differences in social groups, which have been solely explained by biomedical dimensions. Although there have been advances in the biological understanding of these diseases, they do not account for the complexity of the phenomenon. To address this, the introduction of psychosocial variables have shown that they may be useful to understand the manifestations and evolution of these conditions. Thus, gender gaps should not be studied exclusively from a biomedical perspective. This article reviews the explanations about the gender gaps in the expression of acute myocardial infarction based on gender stereotypes, as an alternative to the biomedical approach. Stereotypes can predict different consequences when they are used by health professionals or patients. Finally, alternatives are proposed to reduce the negative results of the gender stereotype activation when an acute myocardial infarction is suffered. Also new development lines in theoretical, methodological and interdisciplinary work are proposed.

(Rev Med Chile 2018; 146: 921-926)

Key words: Discrimination (Psychology); Health Behavior; Myocardial Infarction; Physician-Patient Relations; Stereotyping.

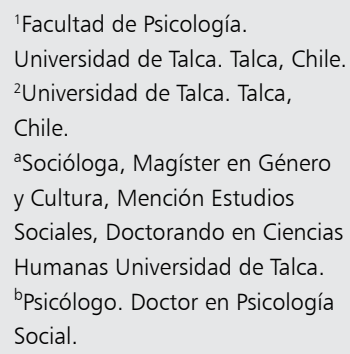

Fuente de Financiamiento: Este trabajo se ha desarrollado con el apoyo del proyecto Fondecyt 1161661 de responsabilidad del segundo autor.

Recibido el 27 de junio de 2018 , aceptado el 16 de agosto de 2018.

Correspondencia a:

María Pía Martínez M.

Universidad de Talca. Talca, Chile.

mamartinez@utalca.cl

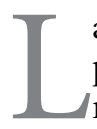

a enfermedad cardiovascular constituye la primera causa de muerte en las mujeres a nivel mundial. Una reciente y exhaustiva revisión de las brechas de género de infarto agudo de miocardio (IAM) en Estados Unidos de Norteamérica ${ }^{1}$ muestra que hombres y mujeres difieren en la patofisiología de la enfermedad (e.g., proporción de personas con ruptura de placas artereoscleróticas) y en su presentación clínica (e.g., proporción de síntomas atípicos). Paradójicamente, se ha encontrado que en las mujeres, con igual carga de factores de riesgo, es menos grave la extensión de la enfermedad coronaria epicárdica ${ }^{1}$. Asimismo, en Chile la enfermedad se manifiesta con mayor frecuencia en hombres, pero a medida que avanza la edad, la mujer se equipara y lo supe$\mathrm{ra}^{2}$. Al igual que en otros países, a su vez, es mayor la letalidad femenina en contexto intrahospitalario y 30 días después del evento ${ }^{1,3,4,5}$.

Pese a los avances en la comprensión de brechas de género en el IAM, existen controversias. Por ejemplo, en la patofisiología de la enfermedad, una de las ideas más extendidas es la que sugiere que la hormona estrógeno es un factor protector $^{4}$. Sin embargo, algunas investigaciones han mostrado que puede no tener efecto alguno e, incluso, relacionarse con un aumento en el riesgo a infartarse $e^{6-8}$.

En relación con la presentación clínica de la enfermedad, estudios muestran que podrían 
existir dificultades de diagnóstico de IAM en mujeres, dado a que se manifiesta con síntomas atípicos o difusos ${ }^{1,5,9,10,11}$, de muy baja ocurrencia en varones ${ }^{12}$. El tratante puede confundir relatos de síntomas de angina con estrés o depresión ${ }^{10}$. Estas dimensiones poco frecuentes pueden tener relación con fenómenos ajenos a la sola diferencia anatómica entre sexos, como, por ejemplo, la construcción social de la misma enfermedad ${ }^{10}$.

Para entender estas discrepancias se han propuesto diferentes alternativas. Algunos autores han señalado que existen problemas metodológicos en los estudios como, por ejemplo, que en algunas investigaciones clínicas las mujeres fueron subrepresentadas ${ }^{1,10,13,14}$ o, que en ciertos trabajos se ha atribuido peso causal a asociaciones entre variables, por ejemplo, asociación IAM-depresión ${ }^{15}$, IAM-estrés ${ }^{16}$ e IAM-conductas no saludables ${ }^{17,18}$. En el marco de esta discusión, se ha llegado a pensar que la enfermedad cardíaca es diferente en la mujer, a tal punto que las herramientas de diagnóstico utilizadas pueden ocultar su nivel de riesgo real ${ }^{1,19}$.

En esta línea, existen explicaciones sobre la diferencia de la enfermedad en la interacción entre características de las personas y su entorno. Diversas investigaciones muestran que miembros de diferentes grupos sociales (e.g., clase social, etnia, sexo) experimentan la enfermedad de manera distinta antes, durante y después de su ingreso al sistema de salud ${ }^{10,20}$. En este sentido, la enfermedad es un fenómeno construido socialmente, en donde su vivencia (como actor u observador) levanta percepciones que son compartidas socialmente ${ }^{21}$, definiendo lo que las personas entienden de ella. Así, es un fenómeno cultural, que permite conectar los significados de individuos y sus comunidades con respecto al proceso de enfermedad-salud y el cuerpo humano ${ }^{22}$. La existencia de estas discusiones pone de manifiesto que el fenómeno del IAM es más complejo que su sola manifestación médica, por tanto, la epidemiología moderna debiese considerar que existen múltiples dimensiones para comprender este fenómeno.

\section{El infarto como enfermedad estereotipada: efecto Yentl y efecto Pigmalión}

Una de las descripciones más extendidas sobre cómo ocurre el infarto, ha llevado a suponer que este tiene, principalmente, características masculinas. A principios de la década 1990-99, Bernardine Healy $^{23}$ sugirió que las diferencias de género que se observan en la realidad social también se reflejan en el IAM. El efecto Yentl (o síndrome de Yentl) es aquel en donde la mujer, para ser diagnosticada de IAM debe cumplir con el biotipo de IAM, definido como masculino. Dicho de otro modo, debe "convertirse en hombre". En su argumento, Healy evidencia con datos empíricos la asociación entre sexo y el uso de cateterización cardíaca y cirugía de bypass, independientemente de otras variables clínicas. También se han registrado diferencias en el proceso diagnóstico y frente a los mismos síntomas, en donde los hombres son más proclives a ser sometidos a pruebas diagnósticas complementarias y a ser diagnosticados de infarto ${ }^{24}$. En contexto de urgencias ${ }^{25}$, se mostró que fueron diagnosticados de IAM más hombres y mujeres que presentaron síntomas típicos tuvieron mayor probabilidad de ser diagnosticadas de IAM que aquellas con atípicos. Situación similar tuvieron las mujeres con una mayor cantidad de síntomas, pero no se registraron diferencias significativas ante la presencia de síntomas típicos ${ }^{11}$. A su vez, el uso de los tratamientos presenta diferencias según el sexo del paciente, las mujeres reciben en menor medida fármacos (aspirina y beta-bloqueadores) y tratamientos invasivos con angioplastia coronaria ${ }^{1}$.

En la actualidad hay evidencia de la vigencia del efecto Yentl $1^{11,26,27}$, puede no estar tan claro cómo se activa el estereotipo de género en el contexto de salud. Los estereotipos son entendidos como creencias sobre las características, capacidades o comportamientos que tiene un grupo social y sus miembros ${ }^{28}$ y pueden ser usados para interpretar una situación en particular, sin necesidad de dedicar demasiado esfuerzo cognitivo en ello. Dado que la enfermedad está definida como masculina, la única manera en que una mujer pueda ser diagnosticada de IAM es que su comportamiento y sintomatología se empareje lo más posible a lo esperado por el perfil. En línea con esta idea, un estudio experimental sobre narrativas de diagnóstico en profesionale ${ }^{29}$ mostró que estos usan el sexo del paciente como un indicador de riesgo de IAM. Con ello, reducen la probabilidad que las mujeres obtengan un diagnóstico acertado.

Una segunda explicación proviene de estudios en educación. Rosenthal y Jacobson (1965) rea- 
lizaron un estudio en que mostraron a un grupo de profesores los resultados de supuestas pruebas de inteligencia de cada uno de sus alumnos. Al cabo de un tiempo, los estudiantes que poseían mayor inteligencia mostraron mejores calificaciones en pruebas estandarizadas de aprendizaje que aquellos estudiantes indicados como "menos inteligentes" 30 . Este resultado podría parecer aparentemente obvio, si no fuera por el hecho de que la definición de quién era o no inteligente no fue determinada por la prueba, sino por el azar. Si, entonces, la inteligencia se distribuye de manera similar entre los grupos, ¿cómo se explica que uno de estos grupos tenga mejores calificaciones? Rosenthal y Jacobson sugirieron que las expectativas de los profesores sobre sus alumnos fueron las que determinaron los cambios en rendimiento. A esta consecuencia se le domina "Efecto Pigmalión" o "Profecía autocumplida". Numerosos estudios confirman que este efecto ocurre en diferentes contextos educativos $^{31}$.

Del mismo modo que en los estudios de Rosenthal, la profecía autocumplida puede operar en un contexto de salud. Dado el perfil masculino de IAM, un profesional puede inferir que una mujer no puede tener IAM, puesto que no espera de ella que así sea. Como consecuencia, puede solicitar exámenes adicionales, realizar diagnósticos errados (dados los síntomas atípicos) ${ }^{32}$, etc. La existencia de los efectos antes descritos implica, para la persona que recibe, diagnostica y trata a un paciente con IAM, estar atento a estos potenciales sesgos y aumentar la dedicación al paciente y sus síntomas. En caso contrario, es muy probable que sea afectado e influido por el contenido social de género, aumentando la probabilidad de errar y acrecentando con ello las brechas entre hombres y mujeres en diagnóstico y tratamiento de la enfermedad.

\section{El efecto de los estereotipos en el paciente: la amenaza del estereotipo}

Los efectos de los estereotipos no solo se pueden observar en las conductas que realiza un profesional sino, también, en las conductas de los mismos pacientes. Las mujeres tienden a demorar más tiempo entre el inicio de los síntomas y la realización de la consulta que los hombres. En ese tiempo, ellas realizan una serie de acciones o justi- ficaciones para evitar la consulta inicial, tales como aludir al respeto y cuidado del tiempo del profesional ${ }^{33,34}$, buscar ayuda en su red de apoyo para reconocer el riesgo de los síntomas percibidos y definir estrategias de resolución (ingerir alimentos o bebidas especiales o esperar a ver si disminuían los síntomas $)^{10,20,33}$, recurrir al historial previo de relaciones problemáticas entre la paciente y algún profesional o aludir a las responsabilidades asociadas al rol de género que impide destinar tiempo para solicitar atención médica (e.g., esperar que llegue su hijo del colegio) $)^{34}$.

Todas estas justificaciones pueden deberse a que el contexto de consulta refleja ciertos atributos de la mujer que son evaluados negativamente por la sociedad y que las mujeres no desean tener. De hecho, en un estudio ${ }^{34}$, la mitad de ellas reconoció que sentía temor de que el especialista las vea como sobrepreocupada, hipocondríaca o ignorante. Esta situación, en donde una persona percibe que un estereotipo negativo (e.g., hipocondríaca) de su grupo de pertenencia (mujer) puede ser aplicado a sí misma se denomina amenaza del estereotipo $(\mathrm{AE})^{28}$. Para que suceda, las personas deben a) reconocer que ser evaluada en función de un estereotipo es algo personalmente importante $y$, b) percibir que dicha característica puede aplicarse a un dominio conductual específico. La investigación ha mostrado que la experiencia de $\mathrm{AE}$ es desagradable, lo que se manifiesta en aumento de los niveles de ansiedad general, presión sanguínea, cortisol, las conductas de sobrealimentación y las de agresión, entre otras ${ }^{34,35}$. Cuando ella ocurre, el desempeño de las personas se ve mermado comparado con personas no amenazadas. Por ejemplo, menor rendimiento de mujeres con $\mathrm{AE}$ que sin $\mathrm{AE}$ en tareas de matemáticas ${ }^{28}$ o peor desempeño al tomar decisiones de gestión, negociación, liderazgo o financieras que mujeres sin $\mathrm{AE}^{36-38}$.

En el contexto de salud, la existencia de AE puede generar dificultades en la comunicación, afectando negativamente la calidad de la atención. Diversas investigaciones ${ }^{10,34,39,40}$ muestran que las narraciones de la enfermedad son diferentes para hombres y mujeres, y que dichas distancias son percibidas por el especialista y por el paciente. Por tanto, si una mujer espera ser tratada de manera diferente por el hecho de ser mujer, entonces puede fallar de manera consciente o inconsciente en la manera de comunicar sus síntomas, para evitar dicho trato. También, algunos trabajos sugieren 
que esta diferencia de discurso ocurre en el trato médico-paciente. Un estudio de Werner y colaboradores $^{39}$ señala que la narración de los síntomas de enfermedad coronaria en la mujer es difusa y poco clara, incluyendo relatos vinculados a lo que se interpreta como histeria o somatización surgidos por una necesidad personal ("desea llamar la atención"). Hedegaard y su equipo ${ }^{40}$ analizaron los relatos entre médico y paciente, observando que existen diferencias en el tipo de conversación en función de lo que realiza (hombre) versus lo que siente (mujer), buscando confirmar mejorías de la enfermedad (hombre) o buscando descontar síntomas (mujeres) y enfatizando el tratamiento en función de lo que se ha mejorado (hombres) versus aquello que no ha mejorado o aparecido como sintomático (mujeres). Estas formas de comunicación pueden afectar a cómo los pacientes se presentan en términos personales y de sintomatología, de modo que esta sea inconsistente con la categoría de juicio y también ocurre en integrantes de grupos estereotipados ${ }^{34}$.

Finalmente, la manera en que hombres y mujeres adhieren a tratamientos y estrategias de rehabilitación es diferente. Esto puede ser entendido como producto de una situación que amenaza la propia identidad. Dado que dicha situación levanta sentimientos de ansiedad e incrementa la rumiación ${ }^{34}$, una mujer podría no entender de manera adecuada la naturaleza e importancia de las recomendaciones sugeridas por el especialista, o tener dificultad para recordar información relevante para dicho tratamiento ${ }^{34}$. Esto podría explicar por qué, luego de egresar del hospital, la mujer tiene indicadores más bajos de adherencia farmacológica y de rehabilitación ${ }^{41,42}$, lo que se asocia a la importancia del rol de género ${ }^{10}$. Por ejemplo, al encuestar pacientes postinfarto y sus parejas sobre la división de tareas domésticas previo y los seis meses posteriores al evento, se reveló que las mujeres infartadas retomaron sus actividades más temprano que sus pares hombres para no descuidarlas ${ }^{43}$. Posiblemente, porque no hacerlo implica dejar de realizar una acción estereotípicamente relevante y que configura su identidad de manera positiva ${ }^{10}$.

\section{Discusión}

Las experiencias aquí presentadas dan cuenta del rol que tiene el estereotipo de género en la toma de decisiones y conductas de pacientes y especialistas. Este abordaje tiene interés metodológico debido a que permite predecir determinado tipo de conductas por parte del paciente (como la demora en acceder al sistema de salud y la no adherencia al autocuidado). Existen instrumentos validados que miden sesgos posibles de validar para el contexto nacional ${ }^{34,44,45}$. A su vez, la presencia de amenaza se manifiesta en cambios fisiológicos medibles ${ }^{46}$. Todo ello contribuye a generar información basada en diseños experimentales involucrando dimensiones biológicas y de identidad individual y social, e invita a la articulación interdisciplinaria.

¿Cómo se pueden evitar las consecuencias negativas de la activación de estereotipos? En el área de educación se ha demostrado que pequeños cambios en patrones conductuales del profesor, tienen un gran impacto en el rendimiento del estudiante $^{34}$. Desde este enfoque, en el área de la salud, algunos han logrado identificar el tipo de representación de la enfermedad que podía predecir la frecuencia de visitas de los pacientes y que, al ser intervenidas, propician un aumento en el número de visitas ${ }^{47}$. En términos amplios, la investigación muestra que una estrategia que busque eliminar o reducir el sentimiento de amenaza, mejora el desempeño y propicia conductas orientadas al cuidado ${ }^{48}$, con ello se vislumbra un camino prometedor para el desarrollo de políticas sanitarias orientadas al cambio conductual.

Una de las principales herramientas para disminuir los sesgos en salud es lograr identificar instancias en las cuales el paciente puede sentir la $\mathrm{AE}$, a partir de una reflexión del profesional y su equipo. Por ejemplo, recordando que los estereotipos operan de manera automática, al interactuar con el paciente, tratar de ponerse en su lugar e identificar posibles temores que puedan interferir en su cuidado ${ }^{49}$. Así, manifestar que existen suficientes evidencias de que la adherencia es relevante para el control de los factores de riesgo $\mathrm{y}$, puede contribuir a mejorar su calidad de vida y reinsertarse en sus actividades. Otra alternativa es por medio de la afectividad positiva, permitiendo el reconocimiento de sus éxitos y avances en su tratamiento, y no solo de la sintomatología de ansiedad y depresión. En último término, no solo es importante intervenir en las personas que pueden ser objeto o sujeto de consecuencias negativas de $\mathrm{AE}$, sino que también cambiar las condiciones que lo hacen posible. 
Estos esfuerzos deberían, entonces, formalizarse en guías terapéuticas y en estudios de investigación experimental, que tengan como objeto promover mejores prácticas clínicas, conocer las creencias asociadas a la enfermedad de la comunidad en la que están trabajando, posibles riesgos de $\mathrm{AE}$ y así definir estrategias que contribuyan a la disminución de su efecto.

\section{Referencias}

1. Mehta LS, Beckie TM, DeVon HA, Grines CL, Krumholz $\mathrm{HM}$, Johnson MN, et al. Acute Myocardial Infarction in women: a scientific statement from the American Heart Association. Circulation 2016; 133: 916-47.

2. Díaz-Toro F, Nazzal C, Verdejo H. Incidencia y letalidad intrahospitalaria por insuficiencia cardiaca en Chile: ¿Existen diferencias por sexo? Rev Med Chile 2017; 145: 703-9.

3. Nazzal C, Frenz PA, Alonso FT, Lanas F. Effective universal health coverage and improved 1-year survival after acute myocardial infarction: the Chilean experience. Health Policy Plann 2016; 31: 700-5.

4. Wenger N. You've Come a Long Way, Baby: Cardiovascular Health and Disease in Women: Problems and Prospects. Circulation 2004; 109: 558-60.

5. Marrugat J, Sala J, Aboal J. Epidemiología de las enfermedades cardiovasculares en la mujer. Rev Esp Cardiol 2006; 59 (3): 74.

6. Arteaga E. Menopausia y riesgo cardiovascular. Rev Med Chile 2016; 132 (6): 761-7.

7. Tindle HA, Chang YF, Kuller LH, Manson JE, Robinson JG, Rosal MC, et al. Optimism, cynical hostility, and incident coronary heart disease and mortality in the Women's Health Initiative. Circulation 2009; 120: 65666.

8. Giltay EJ, Kamphuis MH, Kalmijn S, Zitman FG, Kromhout D. Dispositional optimism and the risk of cardiovascular death: the Zutphen Elderly Study. Arch Intern Med 2006; 166: 431-6.

9. Canto JG, Goldberg RJ, Hand MM, Bonow RO, Sopko G, Pepine CJ, et al. Symptom Presentation of Women with Acute Coronary Syndromes Myth vs Reality. Arch Intern Med 2007; 167 (22): 2405-13.

10. Suls J, Martin R. Heart Disease Occurs in a Biological, Psychological, and Social Matrix: Cardiac Risk Factors, Symptom: Presentation, and Recovery as Illustrative Examples. Ann Behav Med 2011; 41: 164-73.

11. Vaina S, Milkas A, Crysohoou C, Stefanadis C. Coronary artery disease in women: From the Yentl syndrome to contemporary treatment. World J Cardiol 2015; 26; 7 (1): $10-8$.

12. O’Donnell S, McKee G, Mooney M, O’Brien F, Moser Dk. low-onset and fast-onset symptom presentations in acute coronary syndrome (ACS): new perspectives on prehospital delay in patients with ACS. J Emerg Med 2014; 46: 507-15.

13. O’Donnell S, Condell S, Begley C M. Add women \& stir' - the biomedical approach to cardiac research! Eur J Cardiovasc Nur 2004; 3(2): 119-27.

14. Kohler C. Women and medicalization: a new perspective. In Gill LK. Inventing Women: science, technology and gender. Cambridge: Polity Press. Cambridge, Reino Unido. 1992.

15. Paz-Filho G, Luicinio J, Wong ML. Pathophysiological basis of cardiovascular disease and depression: a chicken-and-egg dilemma. Rev Bras Psiquiatr 2010; 32 (2): 181-91.

16. Pascoe EA, Smart L. Perceived Discrimination and Health: A Meta-Analytic Review. Psychol Bull 2009; 135 (4): 531-54.

17. Ortiz M, Willey J, Chiang J. How stress gets under the skin o cómo el estrés psicológico se introduce bajo la piel. Rev Med Chile 2014; 142: 767-74.

18. Corrigan PW, Druss BG, Perlick DA. The Impact of Mental Illness Stigma on Seeking and Participating in Mental Health Care. Psychol Sci Publ Int 2014; 15 (2): 37-70.

19. Varleta P, Concepción R, Julio R, Casanova H, Navarrete C. Ateroesclerosis subclínica en población de riesgo cardiovascular bajo y moderado por Framingham chileno. Rev Med Chile 2016; 144: 30-8.

20. Patel H, Rosengren A, Eckman I. Symptoms in acute coronary syndromes: does sex make a difference? Am Heart J 2004; 148 (1): 27-33.

21. Lefler L. Perceived Risk of Heart Attack: A Function of Gender? Nurs Forum 2004; 39 (2): 18-26.

22. Di Silvestre C. Somatización y percepción subjetiva de la enfermedad. Cinta Moebio 1998; 4: 181-9.

23. Healy B. The Yentl Syndrome. N Engl J Med 1991; 325: 274-6.

24. Martin R. Gender Disparities in the Attribution of Cardiac-Related ymptoms: Contribution of Common Sense Models of Illness. Health Psychol 1998; 17(4): 346-57.

25. Milner K, Funk M, Arnold A, Vaccarino V. Typical Symptoms are predictive of acute coronary syndromes in women. Am Heart J 2002; 31: 283-8.

26. Bairey Merz CN. The Yentl syndrome is alive and well. Eur Heart J 2011; 32 (11): 1313-5.

27. Anderson RD, Pepine CJ. Gender Differences in the 
Treatment for Acute Myocardial Infarction Bias or Biology? Circulation 2007; 115: 823-6.

28. Steele C. A threat in the air: how stereotypes shape intellectual identity and performance. Am Psychol 1997; 56 (2): 613-29.

29. Welch L, Lutfley K, Gerstenberger E, Grace M. Gendered uncertainty and variation in physician's decisions for coronary heart disease: the double-edged sword of "atypical symptoms". J Health Soc Behav 2012; 53 (3): 313-28.

30. Rosenthal R, Jacobson L. Pygmalion in the classroom. Urban Rev 1968; 16-20.

31. Rosenthal R, Jacobson L. Pygmalion in the Classroom: Teacher Expectation and Pupils' Intellectual Development Great Britain: CPI Antony Rowe, Chippenham and Eastbourne; 2003.

32. Mishler E. The discourse of medicine: dialectics of medical interviews. Ablex Publishing Corporation. Norwood, Nueva Jersey, EE.UU. 1985.

33. Schoenberg N, Peters J, Drew E. Unraveling the mysteries of timing: women's perceptions about time to treatment for cardiac symptoms. Soc Sci Med 2003; 56 (2): 271-84.

34. Aronson J, Burguess D, Phelan S, Juarez L. Unhealthy Interactions: The Role of Stereotype Threat in Health Disparities. Am J Public Health 2013; 103 (1): 50-7.

35. Major B, O’Brien L. The social psychology of stigma. Annu. Rev. Psychol 2005; 56: 393-421.

36. Bergeron DM, Block CJ, Echtenkamp A. Disabling the able: Stereotype threat and women's work performance. Hum Perform 2006; 19 (2): 133-58.

37. Burnette JL, Pollack JM, Hoyt CL. Individual differences in implicit theories of leadership ability and self-efficacy: Predicting responses to stereotype threat. J Leadersh Org Stud 2010; 3(4): 46-56.

38. Carr PB, Steele CM. Stereotype threat affects financial decision making. Psychol Sci 2010; 21 (10): 1411-6.

39. Werner A, Isaksen LW, Malterud K. I'm not the kind of women who complains of everything: Illness stories on self and shame in women with chronic pain. Soc Sci Med 2004; 59(5): 1035-45.

40. Hedegaard J, Ahl H, Rovio-Johansson A, Siouta E. Gendered communicative construction of patients in consultation settings. Women Health 2014; 54 (6): 51329.

41. Boogard M, Briody M. Comparison of the rehabilitation of men and women post-myocardial infarction. J Cardiopulm Rehabilitation 1985; 5: 379-84.

42. Di Matteo MR. Social support and patient adherence to medical treatment: a meta-analysis. Health Psychol 2004; 23: p. 207-2018.

43. Rose G, Suls J Green P, Lounsbury P, Gordon E. Comparisons of adjustment, activity, and tangible social support in men and women patients and their spouses during the 6-months post-myocardial infarction. Ann Behav Med 1996; 18: 264-72.

44. Nilsson G, Mooe T, Söderström L, Samuelsson E. Pre-hospital delay in patients with first time myocardial infarction: an observational study in a northern swidish population. BMC Cardiovasc Disor 2016; 16 : 93-103.

45. Hatzenbuehler M, Phelan J, Link B. Stigma as a Fundamental Cause of Population Health Inequalities. Am J Public Health 2013; 103 (5): 813-21.

46. Derks B, Scheepers D, Van LC, Ellemers, N. Reports The threat vs. challenge of car parking for women: How selfand group affirmation affect cardiovascular responses. J Exp Soc Psychol 2011; 47: 178-83.

47. Editorial. The Common-Sense Model of self-regulation of health and illness: how can we use it to understand and respond to our patient's needs? Rheumatology 2007; 46: 904-6.

48. Kit K, Tuokko H, Mateer C. A review of stereotype threat literature and its application in a neurological population. Neuropsychol Rev 2008; 18: 132-48.

49. Ortiz O, Ortiz E. Psicología de la salud: Una clave para comprender el fenómeno de la adherencia terapéutica. Rev Med Chile 2007; 135: 647-52. 